\title{
Comparative analysis of molecular properties and reactions with oxidants for quercetin, catechin, and naringenin
}

\author{
Artem G. Veiko ${ }^{1}$ - Elena A. Lapshina ${ }^{1}$. Ilya B. Zavodnik ${ }^{1}$ (]) \\ Received: 8 May 2021 / Accepted: 10 August 2021 / Published online: 18 August 2021 \\ (c) The Author(s), under exclusive licence to Springer Science+Business Media, LLC, part of Springer Nature 2021
}

\begin{abstract}
Flavonoids, a large group of secondary plant phenolic metabolites, are important natural antioxidants and regulators of cellular redox balance. The present study addressed evaluation of the electronic properties of some flavonoids belonging to different classes such as quercetin (flavonols), catechin (flavanols), and naringenin (flavanones) and their interactions with oxidants in model systems of DPPH reduction, flavonoid autoxidation, and chlorination. According to our ab initio calculations, the high net negative excess charges of the $\mathrm{C}$ rings and the small positive excess charges of the $\mathrm{B}$ rings of quercetin, catechin, and naringenin make these parts of flavonoid molecules attractive for electrophilic attack. The 3'-OH group of the $\mathrm{B}$ ring of quercetin has the highest excess negative charge and the lowest energy of hydrogen atom abstraction for the flavonoids studied. The apparent reaction rate constants $\left(\mathrm{s}^{-1}, 20^{\circ} \mathrm{C}\right)$ and the activation energies $(\mathrm{kJ} / \mathrm{mol})$ of DPPH reduction were $0.34 \pm 0.06$ and $23.0 \pm 2.5$ in the case of quercetin, $0.09 \pm 0.02$ and $32.5 \pm 2.5$ in the case of catechin, respectively. The stoichiometry of the DPPH-flavonoid reaction was 1:1. The activation energies $(\mathrm{kJ} / \mathrm{mol})$ of quercetin and catechin autoxidations were $50.8 \pm 6.1$ and $58.1 \pm 7.2$, respectively. Naringenin was not oxidized by the DPPH radical and air oxygen (autoxidation) and the flavonoids studied effectively prevented $\mathrm{HOCl}$-induced hemolysis due to direct scavenging of hypochlorous acid (flavonoid chlorination). The best antioxidant quercetin had the highest value of HOMO energy, a planar structure and optimal electron orbital delocalization on all the phenolic rings due to the $\mathrm{C} 2=\mathrm{C} 3$ double bond in the $\mathrm{C}$ ring (absent in catechin and naringenin).
\end{abstract}

Keywords Quercetin $\cdot$ Catechin $\cdot$ Naringenin $\cdot$ Structure $\cdot$ Antioxidants $\cdot$ Chlorination

\section{Introduction}

Oxidative stress, a result of overproduction of reactive oxygen/nitrogen/chlorine species and/or impairment in the cellular antioxidative defense system, is considered as an important mechanism of cyto- and geno-toxicity. There is a growing interest in searching for exogenous natural and synthetic antioxidants as therapeutic and pharmacological agents [1-5]. Flavonoids, a large group of secondary plant phenolic metabolites (phenolic acids, phenolic diterpenes, flavonoids, tannins, and coumarins belong to this group), are important natural antioxidants, free radical scavengers, and regulators of cellular redox balance and redox signaling

Ilya B. Zavodnik

zavodnik_i1@mail.ru; zavodnik_ib@grsu.by

1 Department of Biochemistry, Yanka Kupala State University of Grodno, Bulvar Leninskogo Komsomola, 50, 230030 Grodno, Belarus cascades [1-6]. Flavonoids are abundant components of human diet and about 10,000 flavonoids have been found in different natural plant sources. The total amount of flavonoids consumed is estimated at hundreds of $\mathrm{mg} / \mathrm{day}$ [3]. The basic flavonoid structure consists of two phenyl groups joined by a three carbon atom bridge. According to their chemical structures, flavonoids are classified into flavonols, flavones, flavanones, isoflavones, catechins, anthocyanidins, and chalcones.

The molecular structure and mechanisms of antioxidative, cytoprotective, and regulatory activities of flavonoids as well as their potential use (tested both ex vivo and in vivo) have been widely discussed [1-7]. Several mechanisms of action are involved in biological properties of flavonoids such as direct free radical scavenging, transition metal ion chelation, indirect upregulation of cellular antioxidant defense enzymes (such as glutathione S-transferase or UDP-glucuronosyl transferase), activation of survival genes, regulation of a number of signaling pathways and mitochondrial 
function, modulation of inflammatory responses, and antimicrobial action $[5,6]$. Flavonoids interact with many proteins and activate, inhibit, upregulate, or downregulate such cascades as protein kinases, AMPK, MAPK, NF- $k \mathrm{~B}$, and the TGF $\beta$-2/PI3K/AKT pathways, p53-mediated apoptotic events, NF-E2-related transcription factor (NRF2)-mediated activation of genes (the Nrf2-Keap1 pathway), the major regulator of cytoprotective responses to oxidative stress [7, 8]. The flavonoids quercetin, kaempferol, and epicatechin, weak acids of a hydrophobic nature, considerably prevent cellular reactive oxygen species (ROS) production by mitochondria $\left(\mathrm{IC}_{50} \approx 1-2 \mu \mathrm{M}\right)$ and inhibit the redox enzymes, $\mathrm{NAD}(\mathrm{P}) \mathrm{H}$ oxidases, xanthine oxidases, monooxygenases, cyclooxygenases, and lipooxygenases [9].

Direct antiradical/antioxidant capacities of flavonoids are related to redox properties of their readily oxidized phenolic hydroxyl groups and conjugated rings. Bors et al. were the first to claim three structures contributing to the radicalscavenging activity of flavonoids: the 3',4'-ortho-dihydroxyl structure in the $\mathrm{B}$ ring (catechol structure) as a radical target site (a), the $\mathrm{C} 2=\mathrm{C} 3$ double bond with conjugation to the 4-oxo group in the $\mathrm{C}$ ring which is necessary for delocalization of an unpaired electron from the $\mathrm{B}$ ring (b), and the hydroxyl substituent at position 3 of the $\mathrm{C}$ ring which is necessary for enhancement of radical-scavenging activity (c) [10] (Fig. 1). The important role of intramolecular H-bonding was simultaneously claimed (d) [11, 12]. Quercetin, which belongs to the class of flavonols, contains all the four chemical structures that determine high antioxidant activity [13]. In our experiments, the flavonoids considerably inhibited membrane lipid peroxidation (the $\mathrm{IC}_{50}$ values were equal to $9.7 \pm 0.8 \mu \mathrm{M}, 8.8 \pm 0.7 \mu \mathrm{M}$, and $46.8 \pm 4.4 \mu \mathrm{M}$ in the case of quercetin, catechin, and naringenin, respectively) and decreased glutathione oxidation in mitochondria and erythrocytes treated with tert-butyl hydroperoxide [14, 15].

We suggested that the biochemical effects of some flavonoids (quercetin, catechin, and naringenin) were partially connected with modification of the membrane bilayer structure, fluidity, and hydration, as well as with flavonoid ability to regulate mitochondrial membrane permeability and to prevent oxidative stress in membrane compartments [14, 15]. The capacity of flavonoids (e.g., quercetin, baicalin, luteolin, hesperetin, gallocatechin gallate, epigallocatechin gallate, and scutellarein) to inhibit key proteins involved in the coronavirus infective cycle has recently been reviewed [16].

The mechanisms of direct antioxidant activity of polyphenols can be clarified via (1) hydrogen atom transfer (HAT) and proton-coupled electron transfer (PCET) where the proton and electron are simultaneously transferred to the radical in one kinetic step; (2) single electron transfer followed by proton transfer (SET-PT), which is a two-step mechanism); (3) sequential proton loss electron transfer (SPLET), a reverse mechanism with respect to SET-PT which represents relatively stable polyphenol anion formation after the proton loss as the first step of this mechanism; and (4) formation of stable adducts with radicals $[17,18]$. In nonpolar environments (e.g., lipid bilayer), PCET is the only active process of free radical scavenging by flavonoids. Under nonacidic conditions, SPLET is the major mechanism and both the mechanisms take place in reactions against peroxyl or DPPH radicals $[11,19]$. Simultaneously, flavonoids display prooxidant toxic effects associated with ROS generation via the catechol group oxidation to semiquinone radicals and quinones. The semiquinone radicals and quinones arylate protein thiols and form adducts with reduced glutathione [4].

Since electronic and molecular parameters are crucial for biochemical activity, calculations of the structure and thermochemical parameters of flavonoids were widely performed earlier using methods of quantum chemistry [8, 17, 20-22]. Relationships between molecular structures of flavonoids and their biological activities are still not fully understood. The goal of the present study was a comparative analysis of the chemical structures of quercetin, catechin, and naringenin belonging to various flavonoid subclasses (optimal molecular geometry, electronic properties, reactive sites) and their antioxidative and anti-inflammatory activities. Knowledge about flavonoid structures and interactions with different targets seems to be useful to predict biological effects of phytopharmaceuticals.

For analysis of the structure/activity relationships, we compared three flavonoid molecules representing different classes that are most abundant and possess high biochemical activity: quercetin (flavonols), catechin (flavanols or flavan-3-ols), and naringenin (flavanones) (Fig. 1). The chemical structure of lipophilic quercetin is characterized by the catechol $\left(3^{\prime}, 4^{\prime} O\right.$-dihydroxy) group in the $\mathrm{B}$ ring and the double bond in the $\mathrm{C}$ ring between $\mathrm{C}-2$ and $\mathrm{C}-3$ in conjunction with the 4-carbonyl group, as well as the 3-, 5-, and 7-hydroxyl groups. The water-soluble catechin molecule does not have the 2, 3 double bond in conjunction with the 4-carbonyl group in the $\mathrm{C}$ ring and naringenin is a flavanon differing from the quercetin structure in the absence of the $\mathrm{C} 2=\mathrm{C} 3$ double bond in the $\mathrm{C}$ ring and hydroxyl groups at the 3-position on the $\mathrm{C}$ ring and at the 3 '-position on the $\mathrm{B}$ ring (Fig. 1). The parameters of quercetin, catechin, and naringenin molecules extend to a great number of flavonoid subclasses.

In our study, we used methods of theoretical semi- and non-empirical quantum chemical calculations for evaluating the charge distribution in the flavonoid molecules, dipole moments, bond dissociation energies (BDE), and their electronic and molecular parameters that determine the nature of their reactions with radicals. The antioxidative activities of flavonoids were assessed in the model reactions of stable DPPH radical reductions and flavonoid autoxidations 
Fig. 1 Optimized molecular structures of quercetin, catechin, and naringenin (the optimization was performed by the semiempirical AM1 method), calculated excess charges of atoms of flavonoid molecules (using the non-empirical ab initio method with 6-31G basis and UHF method with the Polak-Ribière gradient algorithm)
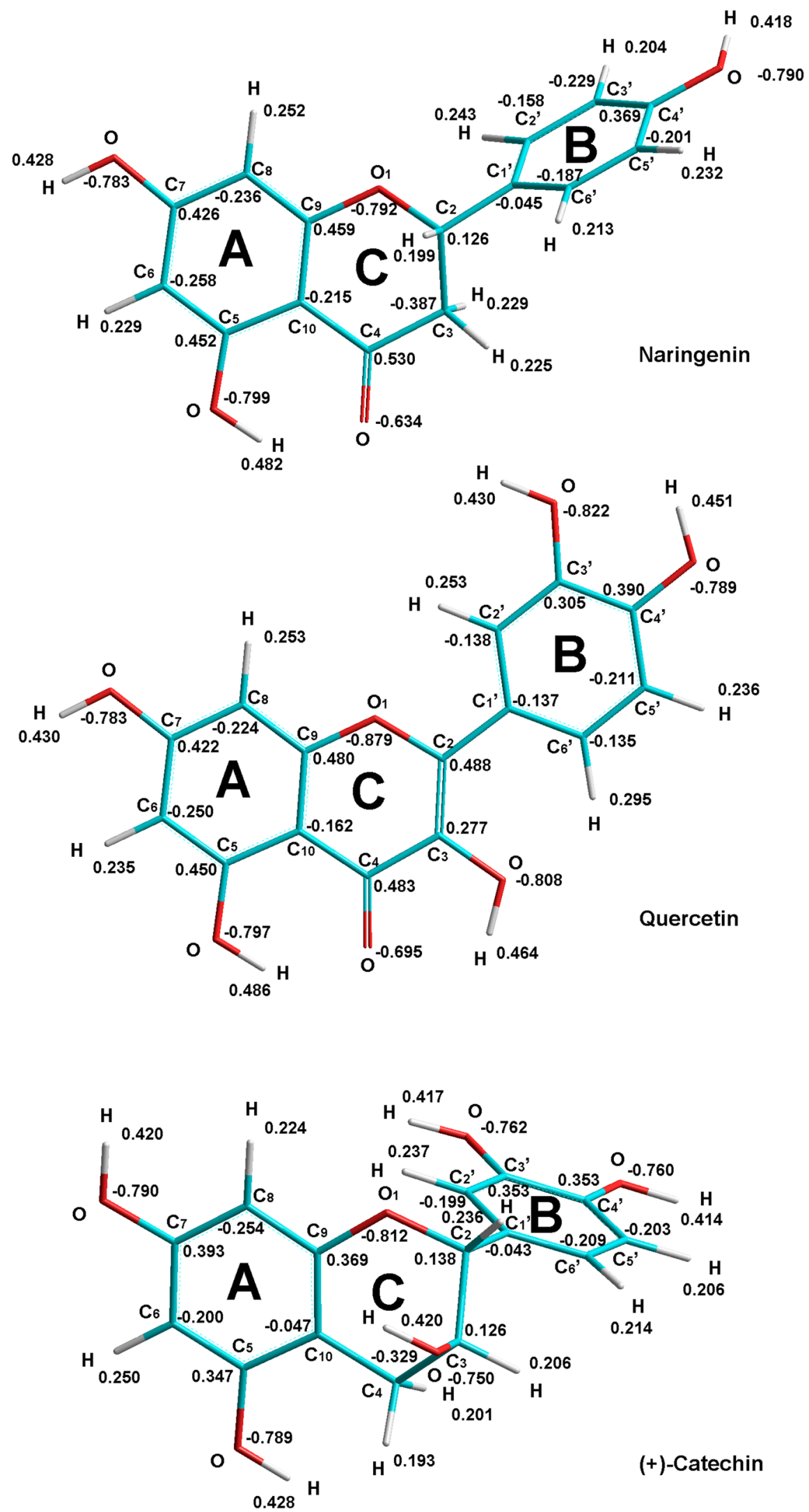
and by a spectroscopy method to measure the reaction rate constants, activation energies, and stoichiometry, using a spectroscopy method. The anti-inflammatory potential of the flavonoids was established as ability to prevent hypochlorous acid-induced red blood cell hemolysis due to flavonoid chlorinations by hypochlorous acid, a dominant mediator of inflammation.

\section{Materials and methods}

\section{Chemicals}

Quercetin, catechin, naringenin, 1,1-diphenyl-2 picrylhydrazyl radical (DPPH), sodium hypochlorite $(\mathrm{NaOCl})$, reduced glutathione, ascorbic acid, melatonin, and other chemicals were from Sigma-Aldrich (St Louis, MO, USA, or Steinheim, Germany). Methanol, ethanol, and other organic solvents and reagents were of analytical grade, purchased from Reakhim (Moscow, Russia), and used without further purification. Flavonoids were used as freshly prepared stock solutions $(15 \mathrm{mM})$ in ethanol. In preliminary experiments, we showed that ethanol at the concentration range used did not affect the parameters measured. All the solutions were made with water purified in the Milli-Q system.

\section{Calculation of flavonoid molecular geometry and electronic properties}

Flavonoid molecules were considered theoretically by performing both the semi-empirical molecular orbital theory and ab initio calculations. The Austin Model 1 (AM1) semiempirical method within unrestricted Hartree-Fock (UHF) formalism in the self-consistent field approximation and the Polak-Ribiere algorithm were considered to optimize fully the geometry of the flavonoid molecules. The electronic structure of the systems was calculated according to an ab initio method $[22,23]$. We performed all the calculations by the HyperChem-8.0 software package (HyperCube, Inc.) for searching the conformations with minimum energy [http://www.hyper.com]. Energy of hydrogen atom abstraction was calculated as the difference of the binding energies of the flavonoid molecule and the corresponding radical formed after removal of hydrogen atom of each hydroxyl group.

\section{Determination of the parameters of DPPH- flavonoid interaction}

The radical-scavenging activities of flavonoids against DPPH stable radical were evaluated by registration of the kinetics of DPPH reduction by flavonoids. Briefly, $10 \mu \mathrm{l}$ of flavonoids at different concentrations (ethanol solutions) was added to $1990 \mu \mathrm{l}$ of a DPPH solution $(100 \mu \mathrm{M}$ in ethanol) at $10-40{ }^{\circ} \mathrm{C}$. The reduction of DPPH was monitored spectrophotometrically as its absorbance change at $520 \mathrm{~nm}$ using a Jasco V-650 UV-VIS Spectrophotometer (Japan). We estimated parameters of DPPH-flavonoid interactions (stoichiometry, apparent rate constants, activation energy) using Eqs. (1) and (2). The DPPH reduction rate equation can be presented as follows:

$v=k c^{n}$,

where $v$ is the apparent reduction rate, $n$ is the order of the reaction, and $k$ is the apparent rate constant of DPPH reduction. The apparent rates of the reduction reactions were estimated from the time dependences of DPPH reduction by varying concentrations of the flavonoids $(2.5-20 \mu \mathrm{M})$. For calculations of the apparent rate constant and the order of the reaction, we represented Eq. (1) in the logarithm form $\ln v=\ln k+n \ln c$. Representing the dependence of the reaction rates on the temperature according to the Arrhenius Eq. (2), we calculated the activation energy of the electron transfer to the DPPH radical:

$v=A \exp (-\Delta E a / R T)$

where $v$ is the rate of the DPPH reduction at the temperature $T(\mathrm{~K}), A$ is the pre-exponential factor, $R$ is the Gas constant, and $\triangle E a$ is the activation energy of the reduction.

\section{Flavonoid autoxidation and chlorination}

Flavonoid autoxidation was monitored spectrophotometrically over $3 \mathrm{~h}$ by recording progressive changes in the absorption spectra during flavonoid $(50 \mu \mathrm{M})$ incubation in open tubes in the dark in ethanol/Na-phosphate buffer, pH 7.4, mixture $(30 \% / 70 \%)$ at $10-50{ }^{\circ} \mathrm{C}$ under gentle stirring. We used phosphate-buffered saline (PBS: $0.15 \mathrm{~mol}^{-1}$ $\mathrm{NaCl}, 1.9 \mathrm{mmol} \mathrm{l}^{-1} \mathrm{KH}_{2} \mathrm{PO}_{4}, 8.1 \mathrm{mmol} 1^{-1} \mathrm{~K}_{2} \mathrm{HPO}_{4}, \mathrm{pH}$ 7.4). Prevention of flavonoid autoxidation by antioxidants was assessed under incubation in the presence of $100 \mu \mathrm{M}$ reduced gluthatione, ascorbic acid, or melatonin.

For chlorination, the flavonoids $(50 \mu \mathrm{M})$ were incubated with $25,50,75,100 \mu \mathrm{M}$ hypochlorous acid $(\mathrm{HOCl})$ in ethanol/Na-phosphate buffer, $\mathrm{pH} 7.4$, mixture $(30 \% / 70 \%)$ for $1 \mathrm{~min}$ at gentle stirring and $25^{\circ} \mathrm{C}$ and the absorption spectra were recorded.

\section{$\mathrm{HOCl}$-induced hemolysis: effects of the flavonoids}

Erythrocytes were isolated from heparinized rat blood by centrifugation (1500 r.p.m., 5 min at $4{ }^{\circ} \mathrm{C}$ ). After removal of plasma and the leukocyte layer, erythrocytes were washed three times with cold $\left(4{ }^{\circ} \mathrm{C}\right)$ phosphate-buffered saline (PBS, pH 7.4). The erythrocytes were used immediately 
after isolation. The care and procedures performed on rats were approved by the Ethics Committee of the Institute of Biochemistry of Biologically Active Compounds, National Academy of Sciences of Belarus (Protocol No. 22/15 of 01.10.2015) and were followed by the National Institute of Health Guide for the Care and Use of Laboratory Animals (NIH Publications No. 8023, revised 1978).

At $\mathrm{pH} 7.4$, the solution of sodium hypochlorite $(\mathrm{NaOCl})$ contained a mixture of $\mathrm{HOCl}$ and $\mathrm{NaOCl}$ at a ratio of approximately $1: 1$ and was subsequently referred to as $\mathrm{HOCl}$ [24]. The susceptibility of erythrocytes to hypochlorous acid-induced damage was measured as the rate of hemolysis. Erythrocyte suspension (hematocrit $0.05 \%$ ) was mixed with varying concentrations of $\mathrm{HOCl}(5-75 \mu \mathrm{M})$ in PBS, $\mathrm{pH}$ 7.4 , at $25^{\circ} \mathrm{C}$, and the kinetic curves of hemolysis were determined spectrophotometrically from the changes in optical density of the suspension at $670 \mathrm{~nm}$ and $25^{\circ} \mathrm{C}$ in the presence or absence of flavonoids. Erythrocyte suspensions had been preincubated with flavonoids for $5 \mathrm{~min}$ before $\mathrm{HOCl}$ was introduced.

\section{Statistical analysis}

The results of the experiments were expressed as the means of four or five replicates \pm SEM. The differences between the values for the parameters measured in groups were analyzed using the Student's $t$ test or the nonparametric Mann-Whitney test depending on the normality of distribution values in the groups. The normality of distribution was determined by the Shapiro-Wilk test. Statistical analysis was conducted using the GraphPad Prism 6.0 software package (https:// www.graphpad.com/quickcalcs). The results were assumed to be statistically significant compared to the control group values when the $p$ value was less than 0.05 .

\section{Results}

For better understanding of the biochemical reactivity of the flavonoids (quercetin, catechin, and naringenin) and their radical-scavenging sites, we compared their structures, properties, and reactions with oxidants.

\section{Molecular properties and geometry of flavonoids}

We calculated the optimized geometry (Fig. 1), molecular, and electronic parameters of quercetin, catechin, and naringenin molecules: heat of formation, dipole moment, surface area, etc. using the semi-empirical molecular orbital theory (Table 1) and excess charges of the atoms of the molecules (Fig. 1) by an ab initio method. The AC and B rings in the quercetin molecule (and not in catechin and naringenin) were located in the same planes (Fig. 1). The torsion angle was $\mathrm{C} 3-\mathrm{C} 2-\mathrm{B} 1^{\prime}-\mathrm{B} 2^{\prime} \approx 180^{\circ}$ due to the double $\mathrm{C} 2=\mathrm{C} 3$ bond in the $\mathrm{C}$ ring of the quercetin structure. Based on the AM1 calculations, the dipole moment of flavonoid molecules increased in the order: quercetin $(0.99 \mathrm{D})<$ naringenin $(1.6$ D) < catechin $(2.1 \mathrm{D})$, which correlates with polarity and water solubility of the flavonoids (Table 1).

According to our ab initio calculations, the net negative excess charges of the $\mathrm{C}$ rings (Table 1 ) of quercetin, catechin, and naringenin make these parts of flavonoid molecules attractive for electrophilic attack. The flavonoid B rings possess positive but small excess charges and the $\mathrm{A}$ rings of the flavonoids demonstrate large positive excess charges. In the quercetin molecule, the calculated HOMO was delocalized mainly on the $\mathrm{B}$ ring and on the $\mathrm{C} 2=\mathrm{C} 3$ double bond and, partially, on the $\mathrm{A}$ and $\mathrm{C}$ rings. In catechin and naringenin molecules, electron orbitals were delocalized mainly on the B ring (Fig. 2).

The calculated highest net negative excess charges were found for hydroxyl groups at the 4'-position in the case of naringenin, at the 3'- and 7-positions in the case of quercetin, and at the 5- and 7-positions in the case of catechin (Table 2). The lowest energies of hydrogen atom abstraction were calculated for the hydroxyl groups at the 4 '-position in the case of naringenin, at the 3-and 3 '-positions in the case of quercetin, and at the 3'- and 4'-positions in the case of catechin (Table 2). We computed the $\mathrm{O}-\mathrm{H}$ bond dissociation energies as the energy evolution associated with the homolytic rupture of the $\mathrm{OH}$ bond [25]. Our present findings are in line with the previous studies. The 4'- and 3'-OH groups (the catechol group) of the ring $\mathrm{B}$ and the $3-\mathrm{OH}$ group of the ring $\mathrm{C}$ were found to be more reactive sites in the quercetin molecule [17]. Participation of the 7-OH group as an orientator of the reaction and not as a direct $\mathrm{H}$-donor in radical scavenging was revealed. The $o$-quinone (for quercetin unhydrate) and $o$-quinone and $p$-quinone (for quercetin dehydrate) derived from the 3'- and 4'-OH groups were suggested to be the main oxidation products of quercetin [17]. It was supposed that the quercetin semiquinone radical arising after removal of the hydrogen atom of the 4'-OH group was the most stable radical and promoted deactivating other radicals via proton and/or electron transfer [18].

\section{Radical-flavonoid interactions}

Figures 3 and 4 represent the dependences of radical reduction rates on the concentrations of flavonoids and the temperature. It should be noted that we recorded the reaction rates under conditions of DPPH excess. Parameters of DPPH-flavonoid interactions (stoichiometry, apparent rate constants, and activation energy) are listed in Table 3. As is seen, the apparent reaction rate constant of the DPPH reduction by quercetin was much higher in comparison with catechin, and naringenin did not demonstrate antioxidant activity. The 
Table 1 Calculated molecular parameters of naringenin, quercetin, and catechin

\begin{tabular}{|c|c|c|c|}
\hline Parameters & Naringenin & Quercetin & $(+)$-Catechin \\
\hline \multicolumn{4}{|l|}{ AM1 UHF } \\
\hline Number of electrons & 102 & 112 & 110 \\
\hline Number of doubly occupied levels & $51 / 51$ & $56 / 56$ & $55 / 55$ \\
\hline Number of total orbitals & 92 & 98 & 98 \\
\hline Total energy, $\mathrm{kcal} / \mathrm{mol}$ & $-85,033.7341$ & $-99,164.7732$ & $-93,070.1819$ \\
\hline Binding energy, $\mathrm{kcal} / \mathrm{mol}$ & -3640.1191 & -3717.6003 & -3861.3785 \\
\hline Isolated atomic energy, $\mathrm{kcal} / \mathrm{mol}$ & $-81,393.6150$ & $-95,447.1729$ & $-89,208.8034$ \\
\hline Electronic energy, $\mathrm{kcal} / \mathrm{mol}$ & $-513,116.2131$ & $-601,021.9335$ & $-597,927.1805$ \\
\hline Core-core interaction $\mathrm{kcal} / \mathrm{mol}$ & $428,082.4789$ & $501,857.1603$ & $504,856.9985$ \\
\hline Heat of formation, $\mathrm{kcal} / \mathrm{mol}$ & -153.7501 & -216.3173 & -211.2465 \\
\hline$E(\mathrm{HOMO}) \alpha, \mathrm{eV}$ & -9.310636 & -8.827891 & -9.042646 \\
\hline$E(\mathrm{HOMO}) \beta, \mathrm{eV}$ & -9.296082 & -8.997514 & -9.061333 \\
\hline$E(\mathrm{LUMO}) \alpha, \mathrm{eV}$ & -0.591512 & -1.085702 & 0.012489 \\
\hline$E(\mathrm{LUMO}) \beta, \mathrm{eV}$ & -0.597053 & -0.852294 & 0.089334 \\
\hline$\Delta E=E(\mathrm{HOMO}) \alpha-E(\mathrm{LUMO}) \alpha, \mathrm{eV}$ & -9.902148 & -7.742189 & -9.055135 \\
\hline$\Delta E=E(\mathrm{HOMO}) \beta-E(\mathrm{LUMO}) \beta, \mathrm{eV}$ & $-9,893,135$ & -8.14522 & -9.150667 \\
\hline Lowest level energy $\alpha, \mathrm{eV}$ & -42.063153 & -42.440206 & -42.205243 \\
\hline Lowest level energy $\beta, \mathrm{eV}$ & -42.057281 & -42.444943 & -42.165635 \\
\hline Highest level energy $\alpha, \mathrm{eV}$ & 6.299569 & 6.340062 & 6.296683 \\
\hline Highest level energy $\beta, \mathrm{eV}$ & 6.299778 & -8.997514 & 6.303410 \\
\hline Dipole moment, D & 1.602 & 0.986 & 2.107 \\
\hline \multicolumn{4}{|l|}{ QSAR properties } \\
\hline Surface area (grid), $\AA^{2}$ & 453.58 & 453.62 & 470.21 \\
\hline Volume, $\AA^{3}$ & 736.63 & 753.52 & 764.33 \\
\hline Hydration energy, $\mathrm{kcal} / \mathrm{mol}$ & -22.97 & -32.53 & -31.12 \\
\hline \multicolumn{4}{|l|}{$\mathrm{Ab}$ initio UHF (6-31G) } \\
\hline Total energy, $\mathrm{kcal} / \mathrm{mol}$ & $-595,475.5297$ & $-688,649.2008$ & $-643,136.5287$ \\
\hline Electronic kinetic energy, $\mathrm{kcal} / \mathrm{mol}$ & $595,905.9009$ & $689,036.7450$ & $643,608.5332$ \\
\hline eK, ee \& eN energy, $\mathrm{kcal} / \mathrm{mol}$ & $-1,516,527.37$ & $-1,770,727.35$ & $-1,704,258.65$ \\
\hline Nuclear repulsion energy, $\mathrm{kcal} / \mathrm{mol}$ & $921,051.8416$ & $1,082,078.1516$ & $1,061,122.1226$ \\
\hline Torsion angles (C3-C2-C1'-C2') & 86.4 & 180 & 117.9 \\
\hline \multicolumn{4}{|l|}{ The total net excess charge of the ring } \\
\hline A & 0.437 & 0.54 & 0.351 \\
\hline $\mathrm{B}$ & 0.069 & 0.128 & 0.018 \\
\hline $\mathrm{C}$ & -0.26 & -0.352 & -0.049 \\
\hline
\end{tabular}

activation energy of DPPH reduction by quercetin was much lower than that of catechin. It means that quercetin is a more preferable reductant of the DPPH molecule than catechin. The stoichiometry of the DPPH reduction was 1:1 (Table 3).

\section{Flavonoid autoxidation: effect of antioxidants}

Flavonoid oxidations by air oxygen (autoxidations) resulted in their spectral changes in time (Figs. 5a, 6, 7a). For quercetin autoxidation, we observed progressive decrease of absorbance maxima at $255 \mathrm{~nm}$ and $378 \mathrm{~nm}$ and appearance of a new peak at $329 \mathrm{~nm}$ (in ethanol/ phosphate buffer mixture, 30\%/70\%, pH 7.4) (Fig. 6a).
Catechin autoxidation resulted in appearance of a new maximum at $435 \mathrm{~nm}$ without significant changes in the peak at $279 \mathrm{~nm}$ (Fig. 7a) and we did not observe spectral changes for naringenin (Fig. 5a). Figure 8 shows kinetic curves of quercetin autoxidation in the absence and in the presence of antioxidants: reduced glutathione, ascorbic acid, and melatonin. From the time dependences of the flavonoid absorbance changes (decrease in $\mathrm{D}_{378}$ in the case of quercetin, and increase in $\mathrm{D}_{435}$ in the case of catechin), we determined the apparent initial autoxidation rates at different temperatures $\left(10-50^{\circ} \mathrm{C}\right)$ and calculated activation energies of the reactions according to the Arrhenius Eq. (2) (Table 3). 
Fig. 2 Calculated HOMO delocalization maps of quercetin, catechin, and naringenin molecules (using the nonempirical ab initio method with 6-31G basis and UHF method with the Polak-Ribière gradient algorithm)
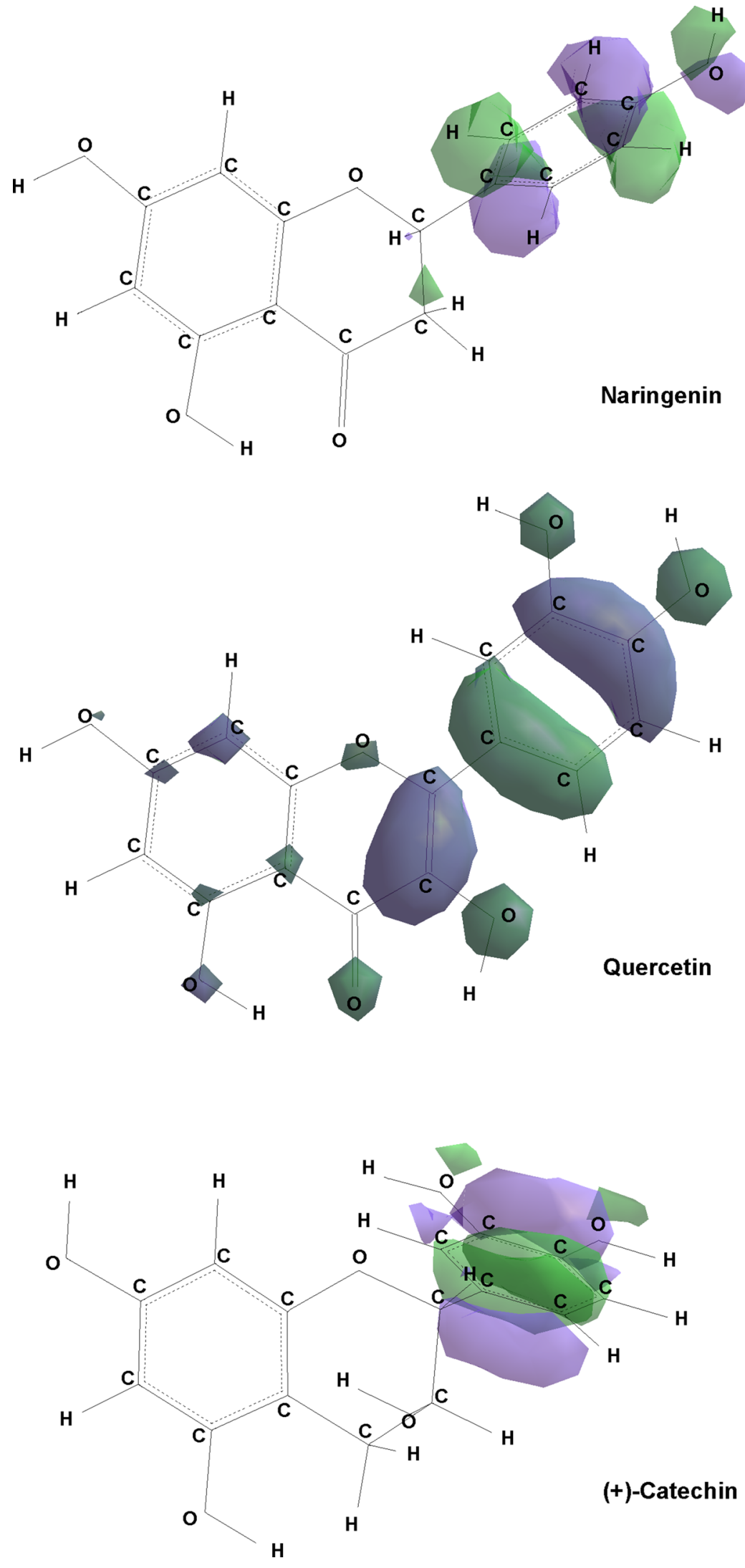
Table 2 Total net excess charge and hydrogen atom removal energy $(\mathrm{kJ} / \mathrm{mol})$ of quercetin, catechin, and naringenin hydroxyl groups

\begin{tabular}{lllllll}
\hline & Hydroxyl group & OH-3 & OH-5 & OH-7 & OH-3' & OH-4' \\
\hline \multirow{2}{*}{ Naringenin } & Bond dissociation energy & - & $-365,59$ & $-352,19$ & - & $-333,35$ \\
& Total net excess charge & - & -0.317 & -0.355 & - & -0.372 \\
Quercetin & Bond dissociation energy & $-309,15$ & $-367,35$ & $-355,63$ & $-316,69$ & $-539,42$ \\
& Total net excess charge & -0.344 & -0.311 & -0.353 & -0.392 & -0.338 \\
\multirow{5}{*}{ Catechin } & Bond dissociation energy & $-435,34$ & $-435,76$ & $-334,85$ & $-317,27$ & $-313,93$ \\
& Total net excess charge & -0.33 & -0.361 & -0.37 & -0.345 & -0.346 \\
\hline
\end{tabular}

\begin{tabular}{|c|c|c|c|c|c|c|}
\hline b & & & $n C$ & & & \\
\hline-13.5 & -13 & -12.5 & -12 & -11.5 & -11 & -10.5 \\
\hline
\end{tabular}

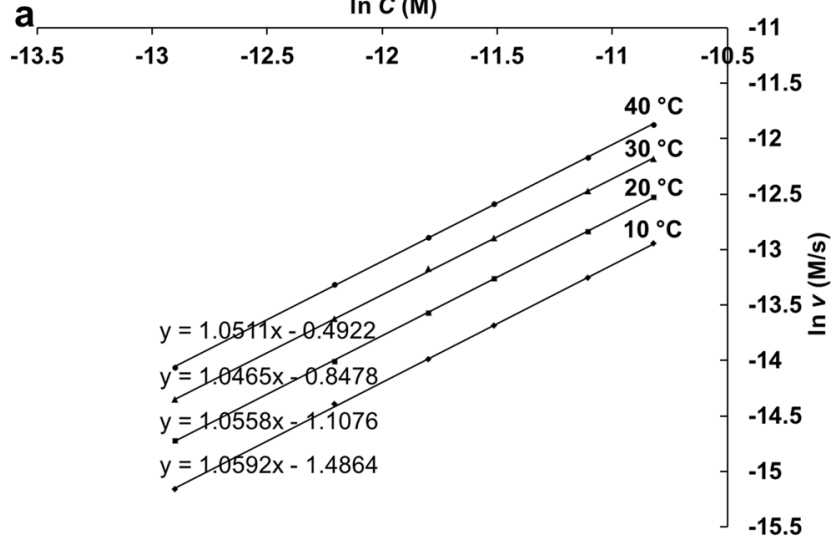

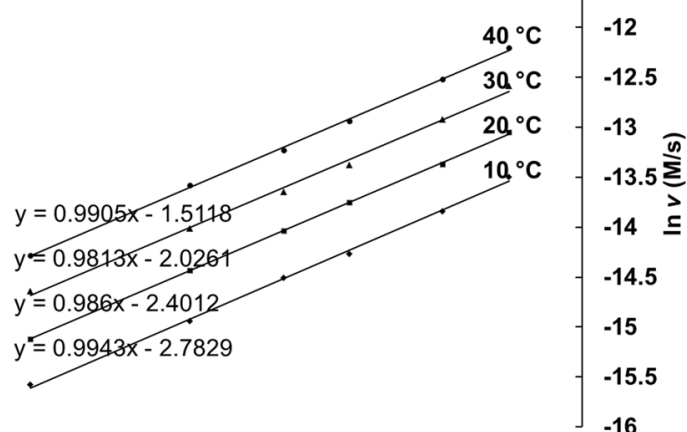

Fig. 3 Concentration dependence of DPPH $(100 \mu \mathrm{M})$ reduction by quercetin (a) and catechin $(\mathbf{b})$ at different temperatures $\left(10-40{ }^{\circ} \mathrm{C}\right)$ in ethanol, represented as the logarithm form

a

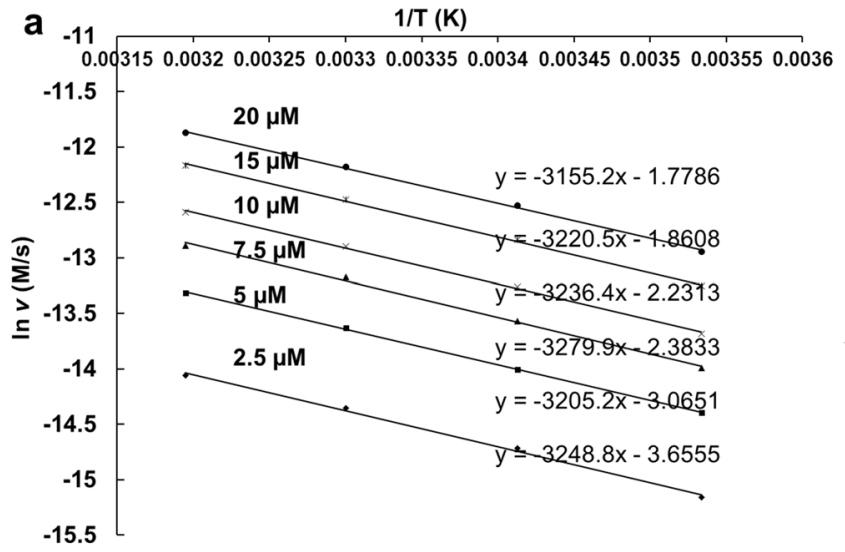

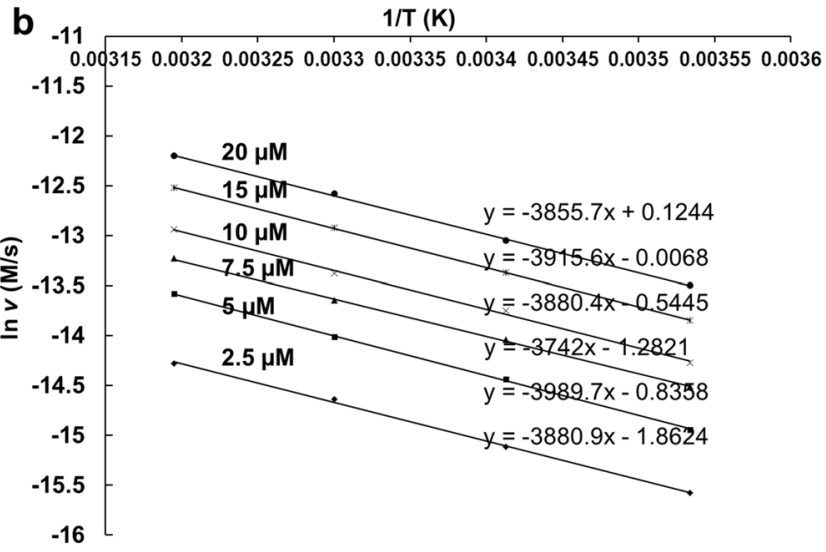

Fig. 4 Arrhenius plots of DPPH $(100 \mu \mathrm{M})$ reduction by quercetin (a) and catechin (b) $(2.5-20 \mu \mathrm{M})$ in ethanol

Table 3 Parameters of flavonoid-oxidant interactions: DPPH reduction, flavonoid autoxidation, inhibition of $\mathrm{HOCl}$-induced hemolysis, and water solubility of flavonoids

\begin{tabular}{llll}
\hline Parameters & Quercetin & Catechin & Naringenin \\
\hline Apparent reaction rate of DPPH reduction, $\mathrm{s}^{-1}\left(20^{\circ} \mathrm{C}\right)$ & $0.34 \pm 0.06$ & $0.09 \pm 0.02$ & - \\
Activation energy of reduction, $\mathrm{kJ} / \mathrm{mol}(10 \mu \mathrm{M}$ flavonoid $)$ & $23.0 \pm 2.5$ & $32.5 \pm 2.5$ & - \\
Reduction stoichiometry & $1.05 \pm 0.05$ & $0.99 \pm 0.05$ & - \\
Activation energy of autoxidation, $\mathrm{kJ} / \mathrm{mol}(50 \mu \mathrm{M}$ flavonoid $)$ & $50.8 \pm 6.1$ & $58.1 \pm 7.2$ & - \\
$\mathrm{IC}_{50}$ for hemolysis inhibition, $\mu \mathrm{M}$ & $1.0 \pm 0.2$ & $1.1 \pm 0.2$ & $1.2 \pm 0.2$ \\
Water solubility, $\mathrm{mg} / \mathrm{l}$ & $0.512[26]$ & $2260[27]$ & $4.38[28]$ \\
\hline
\end{tabular}




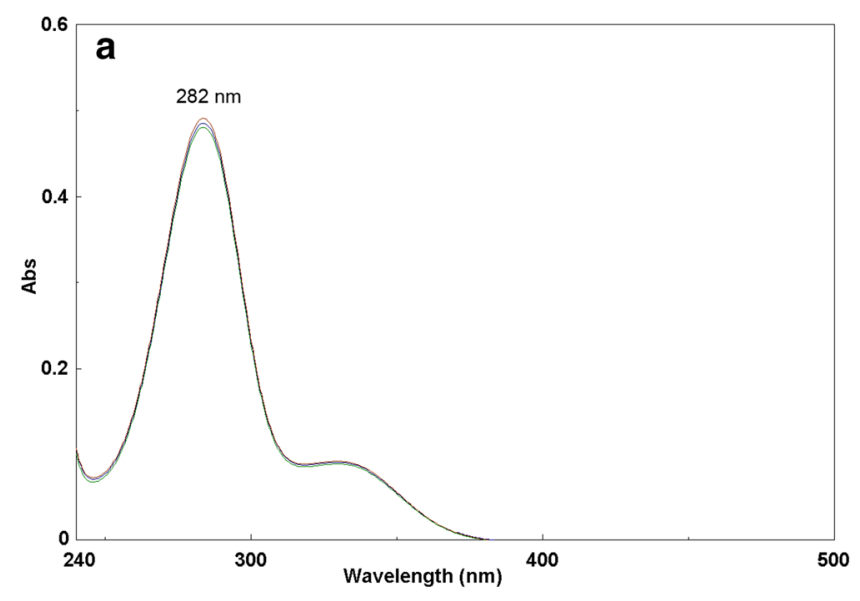

Fig. 5 UV-VIS spectra of naringenin under autoxidation (a) and chlorination (b) (in the presence of $0,25,50,100$, and $150 \mu \mathrm{M}$ $\mathrm{HOCl})$ in ethanol/PBS mixture $(30 \% / 70 \%)$, $\mathrm{pH} 7.4$, the flavonoid con-

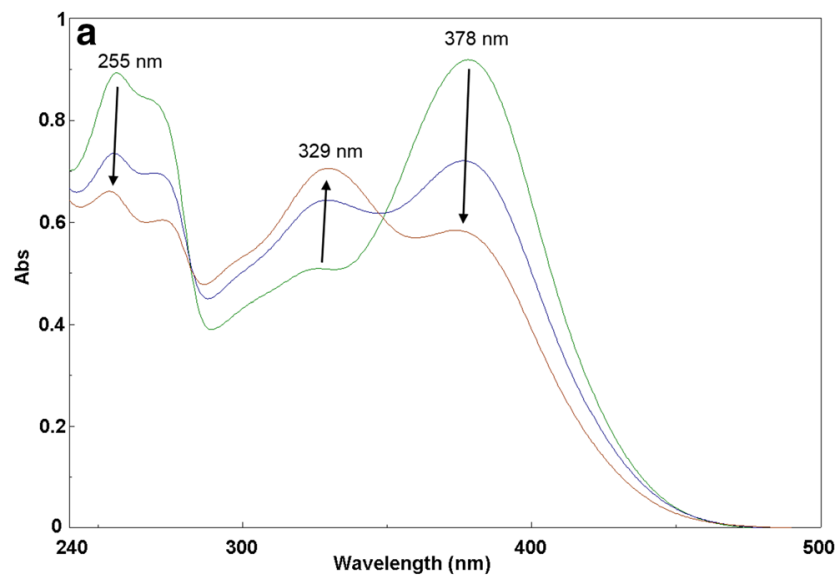

Fig. 6 UV-VIS spectra of quercetin under autoxidation (a) and chlorination (b) (in the presence of $0,25,50,100$, and $150 \mu \mathrm{M} \mathrm{HOCl}$ ) in ethanol/PBS mixture $(30 \% / 70 \%), \mathrm{pH} 7.4$, the flavonoid concentration

All the flavonoids studied effectively interacted with $\mathrm{HOCl}$, a powerful biological oxidant. These interactions (flavonoid chlorination) resulted in considerable changes in the flavonoid absorption spectra (Figs. 5b, 6b, 7b). Spectral changes were similar in the case of quercetin and catechin autoxidation and chlorination, while naringenin chlorination (and not autoxdation) induced a decrease in the peak at $282 \mathrm{~nm}$ and appearance of a new maximum at $370-375 \mathrm{~nm}$ (Fig. 5). Ascorbic acid, reduced glutathione, and not melatonin, prevented flavonoid autoxidation (Fig. 8).

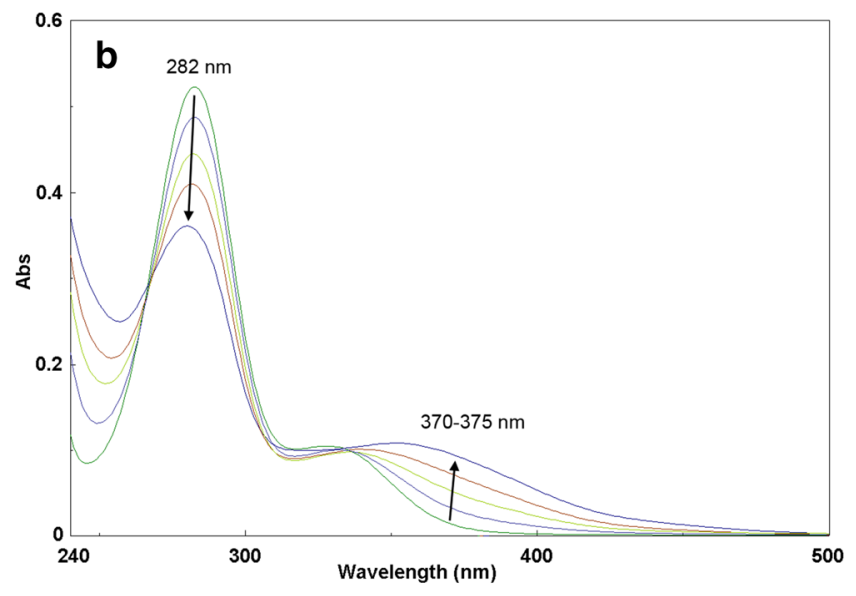

centration was $50 \mu \mathrm{M}$, the temperature of autoxidation was $50{ }^{\circ} \mathrm{C}, 1-\mathrm{h}$ interval, the temperature of chlorination was $25^{\circ} \mathrm{C}, 1 \mathrm{~min}$ of incubation with $\mathrm{HOCl}$

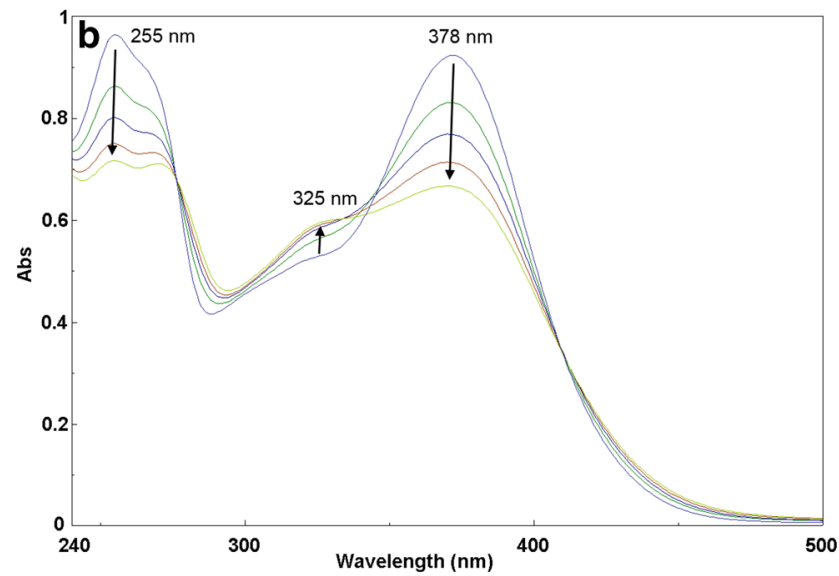

was $50 \mu \mathrm{M}$, the temperature of autoxidation was $50{ }^{\circ} \mathrm{C}, 1$-h interval, the temperature of chlorination was $25^{\circ} \mathrm{C}, 1 \mathrm{~min}$ of incubation with $\mathrm{HOCl}$

\section{Prevention of hypochlorite-induced hemolysis by flavonoids}

The interaction of $\mathrm{HOCl}$ with erythrocytes resulted in effective hemolysis [29]. We assessed the effect of flavonoids on the susceptibility of rat erythrocytes to hypochlorous acidinduced damage, measuring the rates of hemolysis from the kinetic curves of $\mathrm{HOCl}$-induced hemolysis. Flavonoids considerably prevented erythrocyte lysis, as was determined from the dependences of the hemolysis rates on the flavonoid concentrations (Fig. 9). The calculated apparent $\mathrm{IC}_{50}$ concentrations of the flavonoids (Fig. 9, Table 3) for inhibition of the hemolysis were considerably lower than the $\mathrm{HOCl}$ hemolytic concentrations and demonstrated high protective effects of all the flavonoids studied. 


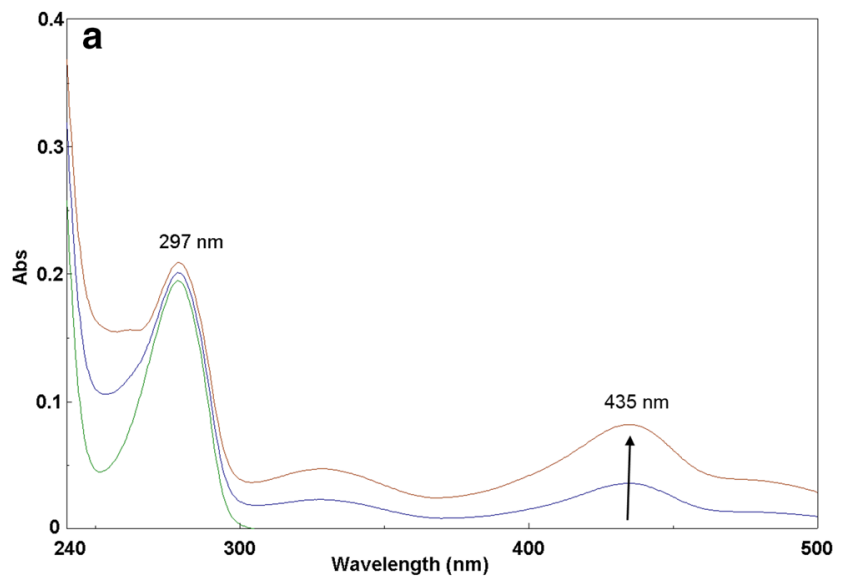

Fig. 7 UV-VIS spectra of catechin under autoxidation (a) and chlorination (b) (in the presence of $0,25,50,100$, and $150 \mu \mathrm{M} \mathrm{HOCl}$ ) in ethanol/PBS mixture (30\%/70\%), $\mathrm{pH} 7.4$, the flavonoid concentration

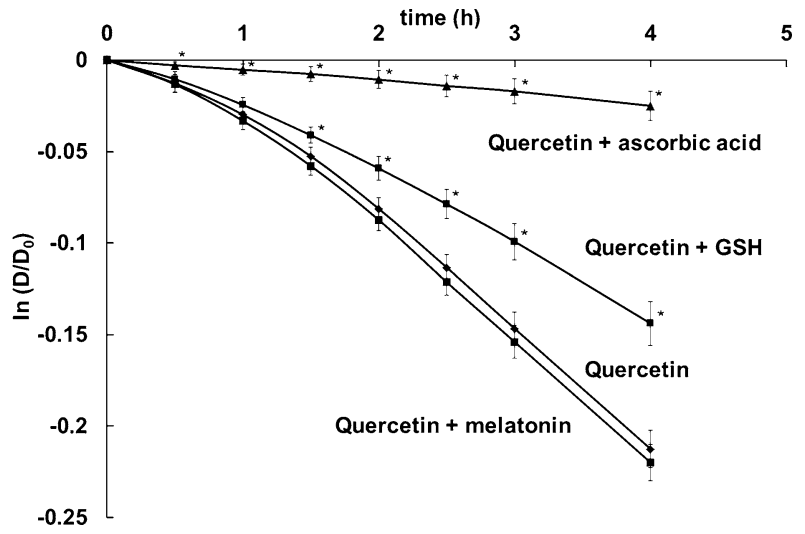

Fig. 8 Time dependences of quercetin absorbance changes $\left(D_{380}\right)$ in the absence of antioxidants, and in the presence of melatonin, reduced glutathione, ascorbic acid in ethanol/PBS mixture (30\%/70\%), $\mathrm{pH} 7.4$, at $25^{\circ} \mathrm{C}$, the flavonoid concentration was $50 \mu \mathrm{M}$, the antioxidant concentrations were $100 \mu \mathrm{M} ;{ }^{*} p<0.05$ in comparison with quercetin alone

\section{Discussion}

The reductive-oxidative reactions of quercetin, catechin, naringenin, their transient and stable oxidation products, as well as the relationship between free radical-scavenging activity of flavonoids and their electronic structure have been widely studied for several decades $[1,9,14-18,22]$. The ability of non-glycosylated flavonoids to function as ROS scavengers is related to the number of hydroxyl groups due to their capacity for hydrogen radical donation and their capability to form stable phenoxyl radicals (a property that is shared by aromatic compounds containing $o$-dihydroxyl groups or close hydroxyl and carbonyl groups) $[9,20,30]$.

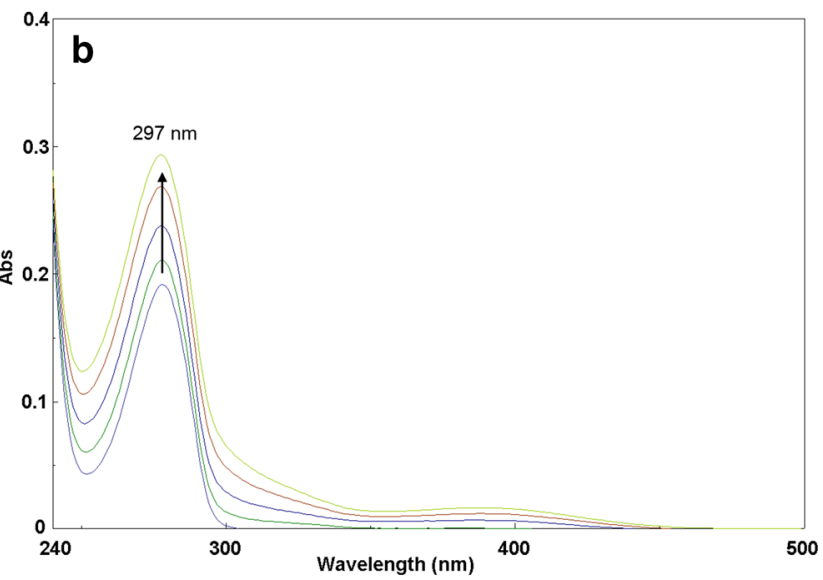

was $50 \mu \mathrm{M}$, the temperature of autoxidation was $50{ }^{\circ} \mathrm{C}, 1$-h interval, the temperature of chlorination was $25^{\circ} \mathrm{C}, 1 \mathrm{~min}$ of incubation with $\mathrm{HOCl}$

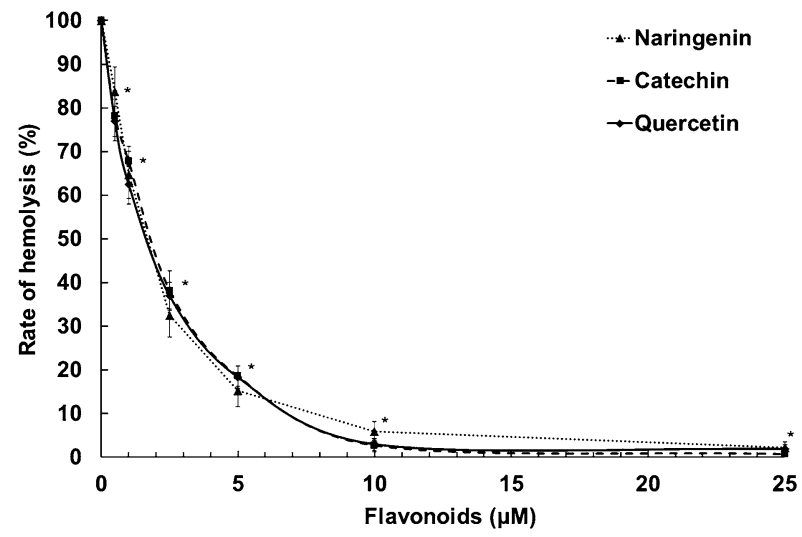

Fig. 9 Inhibition by flavonoids (quercetin, catechin, naringenin) of rat erythrocyte hemolysis induced by $\mathrm{HOCl}$. Erythrocyte suspension (hematocrit $0.05 \%)$ was mixed with $\mathrm{HOCl}(75 \mu \mathrm{M})$ in the absence or the presence of varying concentrations of flavonoids in PBS, $\mathrm{pH} 7.4$, at $25{ }^{\circ} \mathrm{C} ; * p<0.05$ compared with hemolysis in the absence of flavonoids

Flavones and flavan-3-ols are most effective against reactive oxygen species [30].

Forty-eight stable conformers of quercetin have recently been found due to rotation of their five hydroxyl groups and $\mathrm{AC}$ and $\mathrm{B}$ rings and the dipole moment of the conformers varies within the range of 0.35-9.87 D [31]. Earlier, we suggested that the $\mathrm{C} 2=\mathrm{C} 3$ bond in the $\mathrm{C}$ ring determined the planar geometry of quercetin as well as its semiquinone radicals and quinones as optimal forms in vacuum [14]. Similarly, Erkos et al. described stable quercetin conformation as a planar one [22]. The quercetin planar structure (according to our AM1 geometry optimization) is optimal for delocalization of $\mathrm{AB}$ and $\mathrm{C}$ rings electron orbitals (Fig. 2). The catechin and naringenin molecules without the double $\mathrm{C} 2=\mathrm{C} 3$ 
bond in the $\mathrm{C}$ ring did not have any planar structures (Fig. 1, Table 1). On the opposite, it was suggested that in the catechin molecule, an intramolecular hydrogen bond placed the $\mathrm{B}, \mathrm{A}$, and $\mathrm{C}$ rings in the same plane [2]. The dipole moment governs molecule orientation during interaction and interaction rate [22]. According to our calculations, the dipole moments of the highly polarizable $\mathrm{AC}$ ring and the $\mathrm{B}$ ring have opposite directions in the quercetin molecule (data not shown); therefore, quercetin has the lowest dipole moment in comparison with the other two flavonoids, the molecule is non-polar, and possesses low water solubility (Table 1, 3). Earlier, Rasulev et al. calculated the dipole moment for quercetin as $\mu=2.41 \mathrm{D}$ [20], whereas according to Erkos et al., this parameter is $3.013 \mathrm{D}$ [22].

The negative excess charge on the $\mathrm{OH}$ groups as well as on the rings of quercetin and its radicals may play a dominant role in their reactions with radicals and biological activities [22]. The calculated excess negative charges in our experiments allowed to suggest that the $\mathrm{OH}$ groups, located in positions $3^{\prime}$ - and 7-in quercetin, in position 4'in naringenin, and in positions 5- and 7- in catechin, were the most probable sites for hydrogen atom abstraction in radical reduction. Zhu et al. suggested that antioxidative (radical scavenging) activity of flavonoids was directly correlated with the calculated energy of hydrogen abstraction of the phenolic hydroxyl groups (bond dissociation enthalpy, $\mathrm{BDE}$ ) and the order of free radical-scavenging activities of some flavonoids was quercetin $>$ catechin $>$ luteolin $>$ taxifolin $>$ kaempferol $>$ apigenin [1]. The authors showed that the $3^{\prime}-\mathrm{OH}$ and 4'-OH groups possessed the lowest BDEs in the case of quercetin. Similarly, the spin density of the 4'-OH group of quercetin was closely related to its antioxidant activity [1]. The lowest BDEs and the highest excess negative charges of the $3^{\prime}-\mathrm{OH}$ group of quercetin and the $4{ }^{\prime}-\mathrm{OH}$ group of naringenin allow to suggest these groups as the most reactive sites of these flavonoid molecules. It was suggested that the removal of the $\mathrm{H}$-atom on the hydroxyl group on the $\mathrm{C}-4$ ' position of the quercetin $\mathrm{B}$ ring produced the most reactive radical [22].

Our calculation shows that the heat of formation absolute values for the flavonoids increase in the order of naringenin < catechin < quercetin according to molecule stability. On the contrary, it was shown previously that the heat of formation value negatively correlated with the antioxidant activity of polyphenols [32]. The LUMO energy of the flavonoids decreased in the order of catechin $>$ naringenin $>$ quercetin and the HOMO energy was reduced in the order of quercetin $>$ catechin $>$ naringenin (Table 1). Quercetin has the highest value of HOMO energy; thus, quercetin is the best electron donor among the flavonoids studied (possesses the highest reductive potential). Similarly, quercetin has the lowest value of LUMO energy, and it is the best electron acceptor. The regions of the highest density of the
HOMO map (Fig. 2) are preferable for electrophilic attack. The generation of electron density maps in quercetin (and in the quercetin radical) showed that the $\mathrm{C} 2=\mathrm{C} 3$ double bond in the $\mathrm{C}$ ring of quercetin (absent in catechin and naringenin) provides planarity and optimal electron orbital delocalization (HOMO) on all the phenolic rings in the quercetin (and not in catechin and naringenin) molecule (Fig. 2). The system of the conjugated bonds in polyphenol molecules should delocalize electron density and stabilize the transient quercetin semiquinone radicals formed.

In our experiments, the flavonol quercetin was a more powerful antioxidant in comparison with flavan-3-ol (+)-catechin and flavanon naringenin. Quercetin demonstrated much higher apparent reaction rate constant and lower activation energy of DPPH reduction in comparison with catechin, and naringenin did not react with the DPPH radical. One flavonoid molecule was consumed for reduction of one DPPH radical under our experimental conditions (Table 3). It was shown that at excess of flavonoids under the reduction of DPPH, the stoichiometry for flavonoids with one hydroxyl group was 1:1 and that for flavonoids with two hydroxyl groups in the $\mathrm{B}$ ring was 1:2 [33]. The $\mathrm{IC}_{50}$ values for flavonoid interactions with DPPH were determined. They were $5.06 \pm 0.08 \mu \mathrm{M}$ for catechin and $4.36 \pm 0.10 \mu \mathrm{M}$ for quercetin [1]. The activation energy of the DPPH reduction is higher in comparison with the activation energy of diffusion, consequently, not every collision between the reactants would result in DPPH reduction.

Flavonoid autoxidation is one of the main reactions of ROS generation by flavonoids. It was suggested previously that the monoexponential first-order kinetics $\left(k=6.45 \times 10^{-2} \mathrm{M}^{-1} \mathrm{~s}^{-1}\right)$ of quercetin autoxidation, indicating one-electron oxidation of quercetin anion, was followed by two fast steps of radical disproportionation and solvent addition to the resulting quinone. The main products of quercetin autoxidation (as well as enzymatic, chemical, and electrochemical oxidations) are depside, phenolic acids, and quercetin-solvent adducts [34]. The activation energies of quercetin and catechin autoxidation were higher in comparison with that of the flavonoid interaction with DPPH. In our experiments, naringenin did not reduce DPPH and it was not autoxidized, but effectively interacted with hypochlorous acid. The low radical-scavenging activity of naringenin was, probably, connected with the absence of two close hydroxyl groups or the hydroxyl and carbonyl groups in the molecule. Previously, we observed a significant inhibition of membranous lipid peroxidation by naringenin, but its effectiveness was much lower in comparison with quercetin or catechin [14]. The flavonoid autoxidation and chlorination resulted in similar spectral changes although the products of these reactions were different (Figs. 5, 6, 7).

Hypochlorous acid $(\mathrm{HOCl})$ generated from activated neutrophils could be associated with several pathological 
processes such as atherosclerosis. Earlier, we evaluated the sequence of cellular events after red blood cell exposure to HOCl: rapid cell morphological transformations, membrane structural transitions, oxidation of cellular constituents, enzyme modifications, and colloid-osmotic hemolysis [29]. The flavonoids dose dependently prevented $\mathrm{HOCl}$-induced hemolysis and the protective effects of the three flavonoids were similar. We suggested a direct interaction of the flavonoids with hypochlorous acid-flavonoid chlorinations and a decrease of effective $\mathrm{HOCl}$ concentration in erythrocyte suspensions. It is known that the reaction of $\mathrm{HOCl}$ with phenolic compounds occurs through an electrophilic attack on an aromatic carbon at the orthoand para-positions to yield chlorine-substituted products $[35,36]$. Our theoretical calculation demonstrates possible sites for electrophilic attack in the flavonoid molecules (negatively charged C atoms, Fig. 1). Similar reactions of 3-chlorotyrosine and 3,5-dichlorotyrosine formations from the reaction of $\mathrm{HOCl}$ with tyrosine (free or peptide-bound) have been studied and chlorotyrosines have been used as a marker of involvement of $\mathrm{HOCl}$ in the processes [37].

\section{Conclusion}

The best antioxidant quercetin has the highest value of HOMO energy as well as the planar structure and the optimal HOMO delocalization due to the $\mathrm{C} 2=\mathrm{C} 3$ double bond in the $\mathrm{C}$ ring. The catechin and naringenin molecules without the double $\mathrm{C} 2=\mathrm{C} 3$ bond in the $\mathrm{C}$ ring have switched conformations. The net negative excess charges of the $\mathrm{C}$ rings of quercetin, catechin, and naringenin and the small positive excess charges of the $\mathrm{B}$ rings make these parts of the flavonoid molecules attractive for electrophilic attack. The calculated highest net excess charges in the hydroxyl groups at the 4'-position in the case of naringenin, at the 3'- and 7-positions in the case of quercetin, and at the 5- and 7-positions in the case of catechin allowed us to suggest these groups to be the most probable sites for hydrogen atom abstraction in the radical reduction reactions. The $3^{\prime}-\mathrm{OH}$ group of the $\mathrm{B}$ ring of quercetin has the highest excess negative charge and the lowest energy of hydrogen atom abstraction for the flavonoids studied and should be suggested as the most reactive reductive site. The stoichiometry of the DPPH reduction by flavonoids (quercetin, catechin) was $1: 1$. The activation energies of flavonoid autoxidation were higher in comparison with that of the DPPH radical reduction. Naringenin was not autoxidized and it did not reduce DPPH. The flavonoids quercetin (despite low solubility) and catechin (with high water solubility) exerted efficient protective effect against free radicals and oxidants. All the flavonoids studied effectively prevented $\mathrm{HOCl}$-induced hemolysis due to direct scavenging of hypochlorous acid (flavonoid chlorination). In this way, the high antioxidative and anti-inflammantory potentials of the polyphenolic compounds could contribute to numerous health benefits.

Author contributions AGV: Data curation, Visualization, Investigation, Software. EAL: Methodology, Conceptualization, Writing-Original draft preparation. IBZ: Conceptualization, Supervision, Validation, Writing-Reviewing and Editing.

Data availability All the data generated or analyzed during this study have been included in this article.

\section{Declarations}

Conflict of interest The authors declare no conflicts of interest.

Ethical approval The animal experiments were conducted in accordance with the ethical guidelines of the Ethics Committee of the Institute of Biochemistry of Biologically Active Compounds, National Academy of Sciences of Belarus (Protocol No. 22/15 of 01.10.2015) and were followed by the National Institutes of Health Guide for the Care and Use of Laboratory animals (NIH Publications No. 8023, revised 1978).

Informed consent All the authors meet the qualifications for authorship and had an opportunity to read and comment the manuscript. All authors support publication of the manuscript in Molecular and Cellular Biochemistry.

\section{References}

1. Zhu L, Chen J, Tan J, Liu X, Wang B (2017) Flavonoids from Agrimonia pilosa Ledeb: free radical scavenging and DNA oxidative damage protection activities and analysis of bioactivity-structure relationship based on molecular and electronic structures. Molecules 22:195. https://doi.org/10.3390/molecules22030195

2. Dorta DJ, Pigoso AA, Mingatto FE, Rodrigues T, Pestana CR, Uyemura SA, Santos AC, Curti C (2008) Antioxidant activity of flavonoids in isolated mitochondria. Phytother Res 22:1213-1218. https://doi.org/10.1002/ptr.2441

3. Hertog MGL, Feskens EJM, Kromhout D, Hertog MGL, Hollman PCH, Hertog MGL, Katan MB (1993) Dietary antioxidant flavonoids and risk of coronary heart disease: the Zutphen Elderly Study. The Lancet 342:1007-1011. https://doi.org/10.1016/01406736(93)92876-U

4. Boots AW, Haenen GRMM, Bast A (2008) Health effects of quercetin: from antioxidant to nutraceutical. Eur J Pharmacol 585:325-337. https://doi.org/10.1016/j.ejphar.2008.03.008

5. Lü J-M, Lin PH, Yao Q, Chen C (2010) Chemical and molecular mechanisms of antioxidants: experimental approaches and model systems. J Cell Mol Med 14:840-860. https://doi.org/10.1111/j. 1582-4934.2009.00897.x

6. Lago JHG, Toledo-Arruda AC, Mernak M, Barrosa KH, Martins MA, Tibério IFLC, Prado CM (2014) Structure-activity association of flavonoids in lung diseases. Molecules 19:3570-3595. https://doi.org/10.3390/molecules19033570

7. Xu D, Hu M-J, Wang Y-Q, Cui Y-L (2019) Antioxidant activities of quercetin and its complexes for medicinal application. Molecules 24:1123. https://doi.org/10.3390/molecules24061123 
8. Safe S, Jayaraman A, Chapkin RS, Howard M, Mohankumar K, Shrestha R (2021) Flavonoids: structure-function and mechanisms of action and opportunities for drug development. Toxicol Res 37:147-162. https://doi.org/10.1007/s43188-020-00080-Z

9. Lagoa R, Graziani I, Lopez-Sanchez C, Garcia-Martinez V, Gutierrez-Merino C (2011) Complex I and cytochrome C are molecular targets of flavonoids that inhibit hydrogen peroxide production by mitochondria. Biochim Biophys Acta 1807:1562-1572. https:// doi.org/10.1016/j.bbabio.2011.09.022

10. Bors W, Heller W, Michel C, Saran M (1990) Flavonoids as antioxidants: determination of radical-scavenging efficiencies. In: Methods in enzymology. Academic Press, Cambridge, pp 343 355. https://doi.org/10.1016/0076-6879(90)86128-I

11. Di Meo F, Lemaur V, Cornil J, Lazzaroni R, Duroux J-L, Olivier Y, Trouillas P (2013) Free radical scavenging by natural polyphenols: atom versus electron transfer. J Phys Chem A 117:20822092. https://doi.org/10.1021/jp3116319

12. Burda S, Oleszek W (2001) Antioxidant and antiradical activities of flavonoids. J Agric Food Chem 49:2774-2779. https://doi.org/ 10.1021/jf001413m

13. Rice-Evans CA, Miller NJ, Paganga G (1996) Structure-antioxidant activity relationships of flavonoids and phenolic acids. Free Radic Biol Med 20:933-956. https://doi.org/10.1016/08915849(95)02227-9

14. Ilyich TV, Veiko AG, Lapshina EA, Zavodnik IB (2018) Quercetin and its complex with cyclodextrin against oxidative damage of mitochondria and erythrocytes: experimental results in vitro and quantum-chemical calculations. Biophysics 63:537-548. https:// doi.org/10.1134/S0006350918040073

15. Veiko AG, Sekowski S, Lapshina EA, Wilczewska AZ, Markiewicz KH, Zamaraeva M, Zhao H, Zavodnik IB (2020) Flavonoids modulate liposomal membrane structure, regulate mitochondrial membrane permeability and prevent erythrocyte oxidative damage. Biochim Biophys Acta 1862:183442. https://doi.org/10. 1016/j.bbamem.2020.183442

16. Russo M, Moccia S, Spagnuolo C, Tedesco I, Russo GL (2020) Roles of flavonoids against coronavirus infection. Chem Biol Interact 328:109211. https://doi.org/10.1016/j.cbi.2020.109211

17. Mendoza-Wilson AM, Santacruz-Ortega H, Balandrán-Quintana RR (2011) Spectroscopic and computational study of the major oxidation products formed during the reaction of two quercetin conformers with a free radical. Spectrochim Acta A 81:481-488. https://doi.org/10.1016/j.saa.2011.06.041

18. Osorio E, Pérez EG, Areche C, Ruiz LM, Cassels BK, Flórez $\mathrm{E}$, Tiznado W (2013) Why is quercetin a better antioxidant than taxifolin? Theoretical study of mechanisms involving activated forms. J Mol Model 19:2165-2172. https://doi.org/10.1007/ s00894-012-1732-5

19. Dhaouadi Z, Nsangou M, Garrab N, Anouar EH, Marakchi K, Lahmar S (2009) DFT study of the reaction of quercetin with ${ }^{\circ} \mathrm{O}_{2}{ }^{-}$and ${ }^{\circ} \mathrm{OH}$ radicals. J Mol Struct 904:35-42. https://doi.org/ 10.1016/j.theochem.2009.02.034

20. Rasulev BF, Abdullaev ND, Syrov VN, Leszczynski J (2005) A quantitative structure-activity relationship (QSAR) study of the antioxidant activity of flavonoids. QSAR Comb Sci 24:10561065. https://doi.org/10.1002/qsar.200430013

21. Morales J, Günther G, Zanocco AL, Lemp E (2012) Singlet oxygen reactions with flavonoids. A theoretical-experimental study. PLoS ONE 7:e40548. https://doi.org/10.1371/journal.pone.00405 48

22. Erkoç Ş, Erkoç F, Keskin N (2003) Theoretical investigation of quercetin and its radical isomers. J Mol Struct 631:141-146. https://doi.org/10.1016/S0166-1280(03)00237-9

23. Onishi T (2018) Quantum computational chemistry: modelling and calculation for functional materials. Springer, Singapore. https://doi.org/10.1007/978-981-10-5933-9
24. Vissers MC, Carr AC, Chapman AL (1998) Comparison of human red cell lysis by hypochlorous and hypobromous acids: insights into the mechanism of lysis. Biochem J 330:131-138. https://doi. org/10.1042/bj3300131

25. Pérez-González A, Rebollar-Zepeda AM, León-Carmona JR, Galano A (2012) Reactivity indexes and O-H bond dissociation energies of a large series of polyphenols: implications for their free radical scavenging activity. J Mex Chem Soc 56:241-249. https://doi.org/10.29356/jmcs.v56i3.285

26. Strugała P, Tronina T, Huszcza E, Gabrielska J (2017) Bioactivity in vitro of quercetin glycoside obtained in Beauveria bassiana culture and its interaction with liposome membranes. Molecules 22:1520. https://doi.org/10.3390/molecules22091520

27. Srinivas K, King JW, Howard LR, Monrad JK (2010) Solubility of gallic acid, catechin, and protocatechuic acid in subcritical water from (298.75 to 415.85) K. J Chem Eng Data 55:3101-3108. https://doi.org/10.1021/je901097n

28. Wen J, Liu B, Yuan E, Ma Y, Zhu Y (2010) Preparation and physicochemical properties of the complex of naringenin with hydroxypropyl- $\beta$-cyclodextrin. Molecules 15:4401-4407. https:// doi.org/10.3390/molecules15064401

29. Zavodnik IB, Lapshina EA, Zavodnik LB, Soszyński M, Bartosz G, Bryszewska M (2002) Hypochlorous acid-induced oxidative damage of human red blood cells: effects of tert-butyl hydroperoxide and nitrite on the $\mathrm{HOCl}$ reaction with erythrocytes. Bioelectrochemistry 58:127-135. https://doi.org/10.1016/s1567-5394(01) 00126-8

30. Nijveldt RJ, van Nood E, van Hoorn DE, Boelens PG, van Norren K, van Leeuwen PA (2001) Flavonoids: a review of probable mechanisms of action and potential applications. Am J Clin Nutr 74:418-425. https://doi.org/10.1093/ajcn/74.4.418

31. Brovarets' OO, Hovorun DM (2020) Conformational diversity of the quercetin molecule: a quantum-chemical view. J Biomol Struct Dyn 38:2817-2836. https://doi.org/10.1080/07391102. 2019.1656671

32. Meng Q, Yang Z, Jie G, Gao Y, Zhang X, Li W, Li B, Tu Y (2014) Evaluation of antioxidant activity of tea polyphenols by a quantum chemistry calculation method-PM6. J Food Nutr 2:965-972. https://doi.org/10.12691/jfnr-2-12-17

33. Butković V, Klasinc L, Bors W (2004) Kinetic study of flavonoid reactions with stable radicals. J Agric Food Chem 52:2816-2820. https://doi.org/10.1021/jf049880h

34. Zhou A, Sadik OA (2008) Comparative analysis of quercetin oxidation by electrochemical, enzymatic, autoxidation, and free radical generation techniques: a mechanistic study. J Agric Food Chem 56:12081-12091. https://doi.org/10.1021/jf802413v

35. Prasse C, Von Gunten U, Sedlak DL (2020) Chlorination of phenols revisited: unexpected formation of $\alpha, \beta$-unsaturated $C 4$-dicarbonyl ring cleavage products. Environ Sci Technol 54:826-834. https://doi.org/10.1021/acs.est.9b04926

36. Acero JL, Piriou P, Von Gunten U (2005) Kinetics and mechanisms of formation of bromophenols during drinking water chlorination: assessment of taste and odor development. Water Res 39(13):2979-2993. https://doi.org/10.1016/j.watres.2005.04.055

37. Fu S, Wang H, Davies M, Dean R (2000) Reactions of hypochlorous acid with tyrosine and peptidyl-tyrosyl residues give dichlorinated and aldehydic products in addition to 3-chlorotyrosine. $\mathbf{J}$ Biol Chem 275:10851-10858. https://doi.org/10.1074/jbc.275.15. 10851

Publisher's Note Springer Nature remains neutral with regard to jurisdictional claims in published maps and institutional affiliations. 